\title{
Automated Student AsSessment: LABEL Recognition In STEM FIGURES
}

\author{
J. A. Davis \\ MacEwan University \\ davisj80@macewan.ca
}

\begin{abstract}
Visual representations such as Free body diagrams are an important part of solving engineering mechanics problems. Automatic Assessment of these types of images is difficult due to the involvement of multiple object types and to their contextual nature. Using a probabilistic approach, an algorithm was created to automatically categorize groups of characters in labels from images into specific object types including: variables, assignment operators, values, units, or words. Using these categories, the algorithm was then able to determine whether the label was an identifier, a point, a dimension, a variable definition, or an equation. A series of representative test cases were chosen and results found that the current algorithm was able to correctly predict the results of all test cases. The paper discusses each step in detail and provides the resulting probability coefficients for the model.
\end{abstract}

Keywords: Automated Assessment, Label Recognition, STEM Figures

\section{INTRODUCTION}

With continual threats of education budget cuts, the economic sustainability of public education systems is becoming an increasingly important topic [1]. One factor which contributes to this is the cost of hiring teaching assistants to mark student's assignments. One potential solution to this challenge is to have the student's digitally solve and submit assignments on tablets and have computers from secure servers automatically mark them. Aside from the economic benefit, this would allow for better marking consistency between students [2], immediate feedback to students and instructors [3], and a wealth of information which could be used to improve pedagogical methods. This idea is currently incorporated in Learning Management Systems [4] but these types of assessments can be restrictive as they are typically in the form of multiple choice quizzes, short answer or numeric responses which assess the student's final answers as opposed to the process of how the students arrived at them. In addition, it is argued that these types of questions may not assess students at a higher level of Bloom's taxonomy $[5,6]$.

A typical student's submission for a STEM assignment can be broken into five different groups of objects: paragraphs of text, figures, equations, tables, and computer programs. There is a large body of literature on automatic assessment techniques which focus on: essays $[7,8]$, computer programs $[3,9,10]$, and mathematics $[11,12]$. There is, however, little in terms of the automatic assessment of STEM images. These types of images are a particularly important representation as they can provide information on the student's conceptual understanding and may represent the starting point of the problem solving process as in the case of Free Body Diagrams when solving mechanics problems [13]. Automatic assessment of figures is complex since they are contextual; having different meanings in different situations. A classical example is the symbol for a spring in mechanics which is the same symbol as a resistor used in circuits. Some ways to differentiate the symbol can be through labeling in the image with word identifiers (spring or resistor) as well as with properties such as providing the spring constant $(k=5 \mathrm{~N} / \mathrm{m})$ or the resistance of the resistor $(R=10 \mathrm{k} \Omega)$. With properties, both the variable and the units provide information into the context of the symbol used in the image. As a first step in the automatic assessment of images, the goal of this paper was to develop an algorithm to automatically assess labels within images and categorize them into different object types within the context of STEM problems.

\section{SOLUTION METHODOLOGY}

\subsection{Overview}

Given a label from an image, the goal is to break it down into different recognizable objects. Different types of label objects exist in STEM images as shown in Fig. 1. Each label brings into context different objects within the image. For example it provides context to different regions (air vs. ground), points (A vs. B), equations and various dimensions and variables for the problem. Here 
labels are categorized as being either identifiers, points, dimensions, variable definitions, or equations. Examples

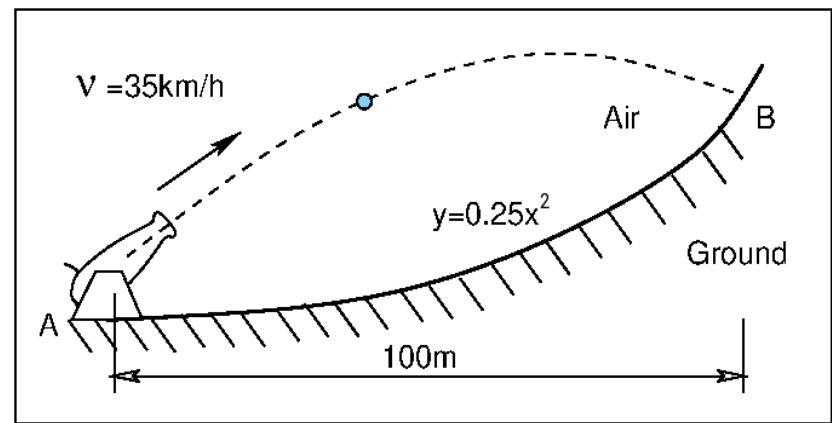

Fig. 1. Example image with various types of labels.

of each label type is shown in Table 1. Each label category can then be broken into specific objects consisting of: variables, spaces, assignment operator, values, units, and words. For example the dimension label " $100 \mathrm{~m}$ " consists of the value " 100 " followed by the unit " $\mathrm{m}$ ". For the current study we consider the recognition

Table 1: Different types of objects in labels

\begin{tabular}{|l|l|}
\hline Label Type & Examples \\
\hline Identifier & Ground, Smooth wall \\
\hline Points & A, B \\
\hline Dimensions & $100 \mathrm{~m}, 35^{\circ}$ \\
\hline Variable Definition & $F=5 \mathrm{~N}, v=35$ \\
\hline Equation & $s=(t+10) \mathrm{m}, y=0.25 x^{2}$ \\
\hline
\end{tabular}

of all of the label types as shown in Table 1 with exception to non-trivial equations which are beyond the scope of this paper. It should be noted that including nontrivial equations would require additional specific objects of type: math and logical operators.

The algorithm used to recognize objects in the labels is shown in Fig. 2. As a starting point to the algorithm, an image is provided in Scalable Vector Graphics format from which the labels can be parsed out from the embedded tags. This type of image format was chosen as it is an open standard, it is based off of Extensible Markup Language (XML) and because XML supports unicode characters of which can be used by students in labeling of STEM images. Ideally, images would be drawn using a stylus and tablet from which standard text and shape recognition libraries would be used to convert the images into SVG format. The programming language used to create and test the label recognition routines was JavaSE 1.7. This language was chosen due to its machine independence, support on Android tablets, widespread use, large availability of libraries, and for its object oriented capabilities. Next, the labels are parsed out from the image. For each label all

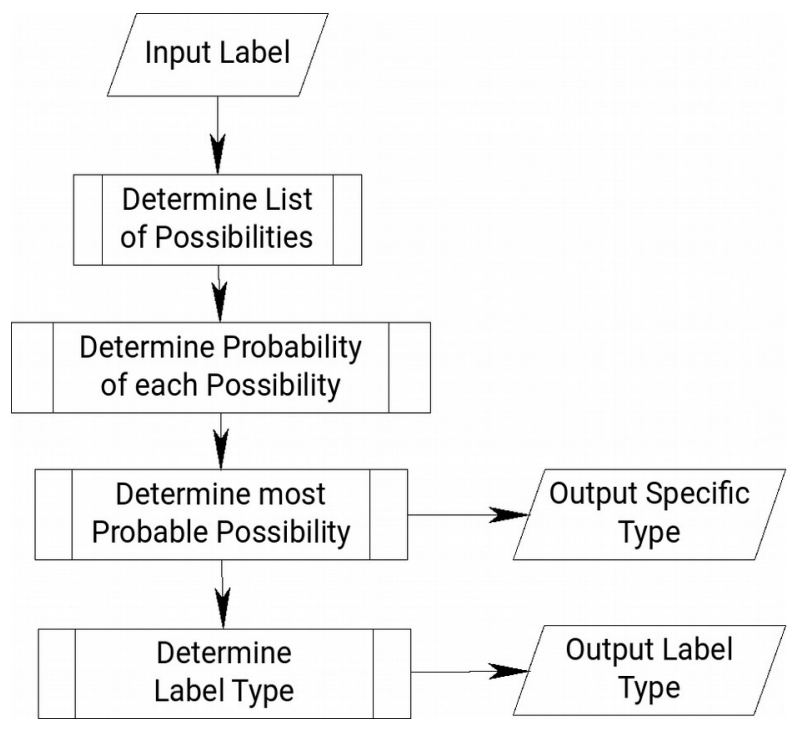

Fig. 2.Diagram showing the label recognition algorithm

possible specific objects (variable, space, assignment operator, value, unit, word) that each character in the label may represent is found. For each possible object, the probability that it is a specific object is then determined (Step 2). The result is then chosen to be the object with the highest probability which is then outputted from the algorithm (Step 3). Knowing the specific types the overall label type is then determined and outputted from the algorithm (Step 4). Each step is further described in the following sections.

\subsubsection{Step 1: Create a list of possibilities}

Given a label, each of these specific object types are found for each character using regular expressions libraries. This technique is similar to chunking or tagging in Natural Language Processing where sentences are broken into different parts of speech categories [6]. Searching the label for each of these specific object types ends up with a list of possibilities. For example, the label

$$
N=5 \mathrm{~m} / \mathrm{s}
$$

would result in a list of possibilities for each character as shown in Table 2. For example, the forward slash

Table 2: List of specific object possibilities for " $N=5 \mathrm{~m} / \mathrm{s}$ "

\begin{tabular}{|l|l|}
\hline Character & Specific Object Possibility \\
\hline$N$ & Variable, Unit \\
\hline$=$ & Assignment operator \\
\hline 5 & Value \\
\hline $\mathrm{m}$ & Variable, Unit \\
\hline$/$ & Unit, Value \\
\hline $\mathrm{s}$ & Variable, Unit \\
\hline
\end{tabular}


character (“/”) could represent either a part of a unit $(\mathrm{m} / \mathrm{s})$ or part of a value $(1 / 2)$.

\subsubsection{Step 2: Probability of each possibility}

The next step is to take the list of possibilities and determine the probability for each possibility. A deterministic approach will not work here since there are potentially multiple solutions within the context of the image and students can potentially make mistakes. Because of this a probabilistic approach is taken. Considering the following label again

$$
N=5 \mathrm{~m} / \mathrm{s}
$$

from Step 1, the character $N$ was found either to be a variable or a unit. So why is it that we recognize $N$ as a variable as opposed to a unit? Some reasons could include: there is typically a value before a unit $(3 \mathrm{~N})$, units usually are placed on the right side of an assignment $(F=3 \mathrm{~N})$, and the resulting equation would be dimensionally unbalanced $(1 \mathrm{~N} \neq 5 \mathrm{~m} / \mathrm{s})$ in the possibility that " $\mathrm{N}$ " is categorized as a unit. It could also be that, in the context of specific image types, force units may not be typically found (meaning that the force unit is unexpected). Next, the character 5 is recognized as a value since (from Step 1) it was found to be the only possibility. Finally, the substring $\mathrm{m} / \mathrm{s}$ could represent multiple possibilities including two variables and a math operator, two units and a math operator, or a single unit. The reason why $\mathrm{m} / \mathrm{s}$ is recognized as a unit could be that in some situations it is an expected unit $(v=5 \mathrm{~m} / \mathrm{s})$, it follows a number (units typically follow values) or it groups the maximum number of similar character types (ie. $\mathrm{m} / \mathrm{s}$ instead of $\mathrm{m}, /, \mathrm{s}$ ). To determine the probability of each possibility, each possibility is passed through a series of tests from which the probability coefficients, $P$, are assigned. This is represented mathematically as

$$
P\left(T_{\mathrm{i}}\right)=\prod_{k} P\left(T_{i} \mid M_{k}\right)
$$

where $P\left(T_{\mathrm{i}}\right)$ is the probability that the character is of object type $\left(T_{i}\right)$ and $P\left(T_{i} \mid M_{k}\right)$ is the probability coefficient for each object type given that the method $\left(M_{k}\right)$ occurs. The tests used to determine the probabilities are shown in Table 3 along with the probability coefficients. It should be noted that initially all probabilities were set to $P\left(T_{i} \mid M_{k}\right)=1$. Most of the methods then set probabilities only when the resultant logical conditions were true for certain specific obect types (ie. if a value was found to be after a unit then the probability coefficient was set to 0.5 ). Other methods set probabilities when the result was both true and false (ie. if an unexpected unit was found the probability was set to 0.1 otherwise it was set to 1.2 ). The groupSimilar() method groups similar specific object types together and removes other possibilities by setting the probability coefficient of neighboring non-similar object types to zero.

Given the label "N=5 m/s" the character " $\mathrm{m}$ " was found to have the possibility of being either a variable or a unit. After running the algorithm the probability that it was a variable was found to be $P$ (variable $)=0.0$ due to the groupSimilar() method which attempts to combine " $\mathrm{m} / \mathrm{s}$ " together. Similarly the probability that it was a unit was $P$ ( unit $)=1.2$ which due to the unexpectedUnits $($ ) method increased the probability.

\subsubsection{Step 3: Determine single possibility}

Given a set of possible types, $T$, for each character in a label, where

$$
T \in\{\text { variable, value, unit, space, asgn. op., word }\}
$$

the most probable type is determined by taking the type with the highest probability.

\subsubsection{Step 4: Determine label type}

Once the most probable specific object types are determined within the label the label type is then determined using logical rules as shown in Table 4. It should be noted that in the event that only a single specific object exists (i.e. the label provided was incomplete and was written as " $\mathrm{m} / \mathrm{s}$ ") the label object will

Table 3: Methods used and their assignment probability

\begin{tabular}{|l|l|l|l|l|l|l|l|}
\hline \multicolumn{1}{c}{ Method } & \multirow{6}{*}{} & \multicolumn{6}{c|}{ Probability Coefficients $\boldsymbol{P}\left(\boldsymbol{T}_{\boldsymbol{i}} \mid \boldsymbol{M}_{\boldsymbol{k}}\right)$} \\
\cline { 3 - 8 } & $\boldsymbol{M}_{\mathbf{k}}$ & Variable & Value & Unit & Space & Asgn Op. & Word \\
\hline valueAfterUnit() & $M_{1}$ & 1.0 & 0.5 & 1.0 & 1.0 & 1.0 & 1.0 \\
\hline unitBeforeValue() & $M_{2}$ & 1.0 & 1.0 & 0.5 & 1.0 & 1.0 & 1.0 \\
\hline unitLeftOfAsgn() & $M_{3}$ & 1.0 & 1.0 & 0.5 & 1.0 & 1.0 & 1.0 \\
\hline unexpectedUnits() & $M_{4}$ & 1.0 & 1.0 & $0.1 / 1.2$ & 1.0 & 1.0 & 1.0 \\
\hline groupSimilar() & $M_{5}$ & 0.0 & 0.0 & 0.0 & 0.0 & 0.0 & 0.0 \\
\hline singleCharacter() & $M_{6}$ & 2.5 & 1.0 & 1.0 & 1.0 & 1.0 & 1.0 \\
\hline words() & $M_{7}$ & 1.0 & 1.0 & 1.0 & 1.0 & 1.0 & 2.0 \\
\hline
\end{tabular}


default to the specific object type. Although this may not occur in correctly drawn STEM images this may result from an error from a student when drawing it.

Table 4: Rules for Categorizing Label Types

\begin{tabular}{|l|l|}
\hline \multicolumn{1}{|c|}{ Label Type } & \multicolumn{1}{c|}{ Logical Rules } \\
\hline Identifier & Words only \\
\hline Points & Single variable only \\
\hline Dimensions & Value and dimension unit \\
\hline Variable Definition & $\begin{array}{l}\text { Variable then contains asgn. op. } \\
\text { then value }\end{array}$ \\
\hline Equation & $\begin{array}{l}\text { Variable then asgn. op. then at } \\
\text { least one more variable }\end{array}$ \\
\hline
\end{tabular}

\section{RESULTS}

In order to test the label categorization algorithm, a series of representative test cases were chosen and tested as shown in Table 5. As an example, the label " $\mathrm{a}=5 \mathrm{~m} / \mathrm{s}^{\wedge} 2$ " was inputted as a label to the algorithm. The output of the specific type from the algorithm was that "a" was found to be variable, "=" was found to be an assignment operator " 5 " was a value, and " $\mathrm{m} / \mathrm{s}^{\wedge} 2$ " was found to be a unit. Using the outputted specific object types the algorithm was able to determine that the inputted label was a "Variable Definition". Both the specific object types and the label type was calculated as expected thus the algorithm passed the given test case. For all test cases the algorithm successfully determined the specific object types within the label and the resulting label type categorization.

\section{DISCUSSION}

The ability to assess specific objects in labels is the first step in recognition of their meaning. Being able to find units or words in labels helps contextualize images such as the example of an image containing a spring or resistor. Given either of the labels " $k=5 \mathrm{~N} / \mathrm{m}$ " or " $R=10 \mathrm{k} \Omega$ " the label assessment algorithm is able to determine that it is a variable definition and then be able to find both the variable and its units from within the label. When combined with a logical statement checking for the type of unit, the algorithm is able to categorize the image as either one from mechanics (spring) or circuits (resistor). Similarly in the case of the label " $\mathrm{N}=5 \mathrm{~m} / \mathrm{s}$ " the algorithm is able to determine that the label is a variable definition and be able to determine the variable and the unit characters. With this information known, a logical step could be created to check the variable and the unit to see if it is as expected (i.e. an expert would expect " $\mathrm{v}=5 \mathrm{~m} / \mathrm{s}$ " as opposed to " $\mathrm{N}=5 \mathrm{~m} / \mathrm{s}$ "). Feedback could then be automatically provided to the student. Another example of the importance is in the assessment of Free Body Diagrams and Kinetic Diagrams as shown in Fig. 3.

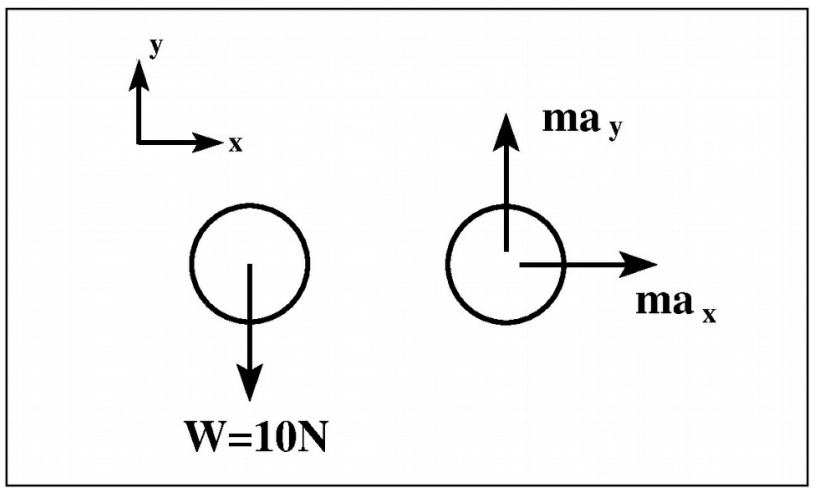

Fig. 3. Example Free Body Diagram and Kinetic Diagram showing labeled vectors

Here multiple arrows are found within the image. As an initial step in assessment of this diagram one must determine whether the arrows represent forces, accelerations, or belong to directions in the axis. The current algorithm will make this possible by contextualizing the labels and allow for the further categorization into the types of forces (such as weight,

Table 5: Test Cases for label recognition algorithm with resulting specific types

\begin{tabular}{|l|l|l|}
\hline \multicolumn{1}{|c|}{ Label Inputted } & \multicolumn{1}{c|}{ Output: Specific object type } & \multicolumn{1}{c|}{ Output: Label Type } \\
\hline $\mathrm{a}=5 \mathrm{~m} / \mathrm{s}^{\wedge} 2$ & var, asgn op, val, unit & Variable Definition \\
\hline$\theta=45^{\circ}$ & var, asgn op, val, unit & Variable Definition \\
\hline$\gamma=45 \alpha$ & var, asgn op, val, var & Equation \\
\hline $5 \mathrm{~km}$ & val, unit & Dimension \\
\hline $\mathrm{N}=2.0 \mathrm{~N}$ & var, asgn op, val, unit & Variable Definition \\
\hline $\mathrm{A}$ & var & Point \\
\hline Smooth Wall & word, word & Identifier \\
\hline $45^{\circ}$ & var, unit & Dimension \\
\hline$-1.5 \mathrm{E} 5$ & val & Value \\
\hline $1 / 12$ & val & Value \\
\hline$\theta=2 \pi \mathrm{rad}$ & var, asgn op, val, unit & Variable definition \\
\hline
\end{tabular}


friction, normal forces) which is necessary when assessing student's work; determining whether there are forces missing, unlabeled, or at an incorrect angle.

\section{CONCLUSIONS}

The assessment of labels is the first step into the automated assessment of images in STEM assignments. An algorithm was created to categorize characters from a label into specific object types variable, assignment operator, value, unit, or word using a probabilistic approach. With this the algorithm was then able to categorize labels into label types (identifier, points, dimensions, variable definition, or equation). A series of representative test cases were chosen and results found that the current algorithm was able to correctly predict the results of all test cases. Discussion of the results show how the assessment of labels in images are useful in the automatic assessment of images in student's assignments in STEM subjects.

\section{References}

[1] S. Sazonov, E. Kharalamova, I. Chekhovskaya, and E. Polyanskaya, "Evaluating Financial Sustainability of Higher Education Institutions," Asian Social Science, vol 11(20), pp 34-40, 2015.

[2] C. Beevers and J. Paterson, "Automatic assessment of problem-solving skills in Mathematics," Active Learning in Higher Education, vol. 4(2), pp 127-144, 2003.

[3] P. Ihantola, T. Ahoniemi, V. Karavirta, and O. Seppala, "Review of Recent Systems for Automatic Assessment of Programming Assignments," Proceedings of Koli Calling ‘10. Koli, Finland, October 28-31, 2010.

[4] A. Trivedi, D. Kar, and H. Patterson-McNeill, "Automatic assignment management and peer evaluation," $J$. of Computing Sciences in Colleges, vol. 18(4), pp 30-37, 2003.

[5] Q. Meng, L. Cutrone, M. Chang, and Kinshuk, "Automarking Web Service," 13th IEEE International
Conference on Advanced Learning Technologies. Los Alamitos, CA: IEEE Computer Society Press, 2013, pp 146-148.

[6] L. Cutrone, M. Chang, and Kinshuk, "Auto-Assessor: Computerized Assessment System for Marking Student's Short-Answers Automatically," Proceedings of the 2011 IEEE International Conference on Technology for Education, India, 2011.

[7] S. Valenti, F. Neri, and R. Cucchiarelli, "An overview of current research on automated essay grading," $J$. of Information Technology Education, vol. 2, pp 319-330, 2003.

[8] L. Rudner, and T. Liang, "Automated Essay Scoring Using Bayes' Theorem," J. of Technology, Learning, and Assessment, vol. 1(2), pp 1-22, 2002.

[9] K. Ala-Mutka, "A survey of automated assessment approaches for programming assignments," Computer Science Education, vol. 15(2), pp 83-102, 2005.

[10] C. Douce, Livingstone, D., and J. Orwell, "Automatic testbased assessment of programming: A review," J. Educ. Resour. Computing, vol. 5(3), pp 1-13, 2005.

[11] P. Kajetanowicz and J. Wierzejewski, "Automatic assessment of math skills," Proceedings of the 7th International Conference Virtual University VU, 2006. pp. 43-47.

[12] C. Beevers and J. Paterson, "Automatic assessment of problem-solving skills in Mathematics," Active Learning in Higher Education, vol. 4(2), pp 127-144, 2003.

[13] P. Kohl, D. Rosengrant, and N. Finkelstein, "Strongly and weakly directed approaches to teaching multiple representation use in physics," Physics Education Research, vol. 3, pp 1-10, 2007. 